\title{
EL INTERNAMIENTO DE EXTRANJEROS: ¿QUÉ DICEN LOS DATOS? \\ ANÁLISIS DE LOS EXPEDIENTES DE \\ INTERNAMIENTO TRAMITADOS EN LA PROVINCIA \\ DE BARCELONA EN EL AÑO 2015
}

\section{FOREIGNER'S PRE-REMOVAL DETENTION: WHAT CAN WE LEARN FROM DATA? ANALYSIS OF PRE-REMOVAL FILES PROCESSED IN THE PROVINCE OF BARCELONA IN 2015}

Markus González Beilfuss, Barak Kalir y Joan-Josep Vallbé

Resumen: El trabajo presenta las principales conclusiones de una investigación empírica sobre los expedientes de internamiento tramitados en la provincia de Barcelona en el año 2015. A partir del análisis de 575 expedientes y del uso de una metodología innovadora, se arroja luz sobre el perfil de las personas extranjeras afectadas por el internamiento, así como sobre los criterios que emplean los diversos agentes que intervienen en esta decisión: policias, fiscales, abogados y jueces de instrucción. También se ofrece información sobre la detención que precede a la solicitud de internamiento y sobre las órdenes de salida obligatoria que se pretende garantizar mediante esta forma de privación de libertad. Todos estos datos permiten poner de relieve cuál es el funcionamiento real del internamiento y los criterios que se emplean en la aplicación de la normativa vigente.

Palabras clave: Internamiento de extranjeros; CIE; inmigración irregular; expulsión de extranjeros; España. 
Summary: This paper contains the most important findings of an empirical research on the detention files of undocumented migrants for the purpose of removal processed in the province of Barcelona in 2015. The analysis of 575 files with an innovative methodology offers relevant information about the profiles of deportable migrants and the way in which all involved actors (police, state prosecutors, lawyers and judges) behave. The study gives also information about the detention that precedes all files, as well as about the deportation order that should be executed thanks to pre-removal detention. All these figures show which is the way in which pre-removal detention works and which are the criteria that are used in the implementation of the legal rules that govern preremoval detention of undocumented migrants.

Key words: Foreigner's detention; detention centres; illegal migration; deportation of foreigners; Spain.

\section{INTRODUCCIÓN}

El internamiento de personas extranjeras en situación irregular ha centrado desde hace tiempo la atención de académicos de diversas disciplinas ${ }^{1}$. Bastantes estudios han permitido conocer mejor cómo funciona el internamiento, cómo son las instalaciones en que se desarrolla esta medida de privación de libertad y cuáles son sus consecuencias. Pero un aspecto que sigue siendo poco tratado es el análisis de los procesos de decisión de los distintos actores que intervienen en esta medida.

Las decisiones sobre el internamiento se toman en entornos de incertidumbre considerable: los diversos actores deben actuar en plazos breves y a partir de informaciones incompletas. La literatura empírica muestra que, en este tipo de circunstancias, los diversos

1 En el caso español, el internamiento y los centros de internamiento (CIE) han sido abordados, entre otros, por González BeIlfuss, M. (2017 y 2016), García España, E. (2017), Boza Martínez, D. (2017), Martínez Escamilla, M. (2016 y 2015), Fernández Bessa, C. y Brandáriz García, J. A. (2016), Daunis Rodríguez, A. (2016), López Benítez, M. (2014), Tomé García, J. (2014), Solanes Corella, A. (2016) y Jarrín Morán, A., Rodríguez García, D. y De Lucas, J. (2013). De la situación de otros países y de los regímenes de deportación en general dan cuenta autores como Kalir, B. (2015 y 2017), KaliR, B. y WissinK, L. (2016), Kalir, B. y Rozakou, K. (2016), Bosworth, M. (2014), Fischer, N. (2012), De Genova, N. y Pautz, N. (2010), Welch, M. y Schuster, L. (2005) y Gibney, M. J. y Hansen, R. (2003), entre otros. 
actores utilizan heurísticos rápidos y frugales ${ }^{2}$, es decir, piezas de información clave que les ayudan a adoptar decisiones con rapidez.

Los estudios sobre la decisión de internar a personas extranjeras y sobre los criterios que se utilizan en la adopción de esta decisión se han visto dificultados por los problemas para acceder y sistematizar la información contenida en los expedientes de internamiento. En España, algunos trabajos recientes han analizado los procesos de toma de decisión en el ámbito de las expulsiones y el internamiento ${ }^{3}$. No obstante, en este último caso subsisten numerosos interrogantes en torno al proceso decisional de esta medida y su funcionamiento real.

El presente trabajo pretende incrementar el conocimiento sobre esta cuestión a través del análisis descriptivo de todos los expedientes de internamiento tramitados en la provincia de Barcelona en el año 2015. Nuestras preguntas iniciales de investigación eran las siguientes: ¿cuál es el perfil de las personas extranjeras afectadas por los expedientes de internamiento? ¿Qué características tienen las solicitudes policiales de internamiento? ¿Cómo motivan los cuatro principales actores implicados en los expedientes de internamiento (policía, fiscales, abogados y jueces) sus respectivas posiciones?

A continuación, se hará alusión a la principal literatura empírica sobre internamiento de personas extranjeras al objeto de formular las preguntas específicas que pretendemos responder. Después, se describirá la regulación del internamiento en España y el papel de los diversos agentes que intervienen en el mismo. Por último, tras describir el origen de los datos y la metodología empleada, se expondrán los principales resultados de la investigación.

\section{LITERATURA Y OBJETIVOS}

La bibliografía española sobre el internamiento apenas se ha centrado en el proceso de toma de decisión de los diversos actores.

2 Gigerenzer, G. y Goldstein, D. G. (1996), Dhami, M. K. y Ayton, P. (2001), y RYo, E. (2016)

3 El reciente trabajo de Barbero, I., Blanco, C. y Arrese, N. (2018) analiza desde una perspectiva cuantitativa los procedimientos de internamiento tramitados en el País Vasco, adaptando la base de datos diseñada en la presente investigación al tipo de datos a los que se tuvo acceso en esta Comunidad Autónoma. Por su parte, los trabajos de Sainz de la Maza Quintanal, E. (2017 y 2015) también son precursores en el empleo de metodologías cuantitativas en el análisis del proceso de expulsión de las personas extranjeras de España. 
En otros países, el estudio empírico de la toma de decisiones judiciales se ha centrado en el uso de heurísticos ${ }^{4}$, aunque muy pocos trabajos lo han hecho en relación con el internamiento. Pero la escasa literatura existente ofrece un precedente interesante para nuestra investigación. Así, en un estudio sobre decisiones acerca de la asignación de fianza en casos de internamiento por parte de tribunales británicos se observó que los jueces tienden a utilizar muy pocos heurísticos (de hecho, solo uno, relacionado con los antecedentes penales de la persona extranjera), y que las resoluciones judiciales dependen mucho de decisiones previas de otros actores como los fiscales y la policía ${ }^{5}$. Por su parte, Ryo ha explorado recientemente el papel de los heurísticos en las vistas sobre fianzas en tribunales de extranjería de Estados Unidos ${ }^{6}$. Ninguno de estos estudios, sin embargo, ha concluido que las características personales de los extranjeros incidan en las decisiones judiciales.

Nuestro trabajo pretende contribuir a la escasa literatura preexistente en dos aspectos. En primer lugar, se trata de un trabajo que no toma como objeto de análisis un sistema jurídico anglosajón, sino uno continental. En segundo lugar, nuestra investigación no se limita a analizar las decisiones de los jueces, sino que engloba a todos los actores relevantes en los procesos de internamiento.

Así, en el caso de la policía nuestro interés se centra en analizar, en primer lugar, qué unidades, de qué modo y con qué argumentos se solicita el internamiento de personas extranjeras; en segundo lugar, qué órdenes de expulsión que se pretende garantizar con esta medida y si existe una selección o priorización de las mismas; y, finalmente, dónde se producen las detenciones policiales que preceden al internamiento.

En el caso de los fiscales nuestra atención se centra no solo en su posición frente a la solicitud policial de internamiento, sino también en los argumentos utilizados para motivarla.

Por lo que respecta a la asistencia letrada de las personas extranjeras, nuestro interés principal radica en el comportamiento de los/las abogados/as durante la comparecencia previa a la decisión judicial sobre el internamiento.

4 Guthrie, C., Rachlinski, J. J. y Wistrich, A. J. (2000); Bainbridge, S. M. y Gulati, G. (2002); Gigerenzer, G. y Engel, E. (2006); Rachlinski, J. J., Wistrich, A. J. y Guthrie, C. (2012).

5 Dhamni, M. K. y Ayton, P. (2001) y Dhami, M. K. (2003).

6 Ryo, E. (2016). 
Por último y en relación con los jueces que toman esta decisión, nuestro foco de atención se ha puesto en los argumentos empleados para autorizar o denegar el internamiento, así como en la posible existencia de diferencias de criterio entre los diversos jueces.

\section{LA REGULACIÓN DEL INTERNAMIENTO Y LOS DATOS OFICIALES SOBRE ESTA INSTITUCIÓN}

La decisión de internar a una persona extranjera en España exige la tramitación de un expediente en el que siempre intervienen los siguientes agentes: funcionarios del Cuerpo Nacional de Policía, representantes del Ministerio Fiscal, la persona afectada con su abogado/a, así como el/la juez/a de instrucción que toma la decisión final. El internamiento es una medida judicial que se se adopta tras un proceso complejo, regulado por diversas normas, circulares e instrucciones, que otorgan a cada actor una función y unos criterios de actuación concretos ${ }^{7}$.

A los funcionarios del Cuerpo Nacional de Policía solo les compete solicitar el internamiento de una persona extranjera si existe una orden de expulsión previa o si se ha iniciado un procedimiento sancionador que puede concluir con una orden de expulsión. La normativa vigente también permite solicitar el internamiento en otros supuestos ${ }^{8}$. Pero la solicitud policial debe tener en cuenta que no estamos ante una sanción, sino ante una medida cautelar que debe tener como única función asegurar la presencia de la persona extranjera para poder ejecutar la expulsión en el plazo máximo de 60 días.

7 La regulación principal del internamiento se encuentra en los art. 62.1 de la Ley Orgánica 4/2000, de 11 de enero, sobre derechos y libertades de los extranjeros en España y su integración social, y 258 del Reglamento que la desarrolla, aprobado por Real Decreto 557/2011, de 20 de abril. La actuación de la policía y los fiscales también se encuentra condicionada por la Circular 6/2014 de la Dirección General de la Policía sobre criterios para solicitar el ingreso de ciudadanos extranjeros en los centros de internamiento y por la Instrucción de la Fiscalía General del Estado 2/2006, respectivamente.

8 Así por ejemplo, cuando se quiere asegurar el cumplimiento de una orden de devolución por haber sido interceptado intentando entrar irregularmente en España o por haber vulnerado una prohibición de entrada (art. 58.6 de la Ley Orgánica 4/2000), así como cuando no se puede ejecutar en 72 horas un rechazo en frontera por incumplir los requisitos de entrada (art. 60 de la misma Ley). 
Los fiscales deben velar por la legalidad de la medida de internamiento, informando la solicitud policial por escrito $\mathrm{y}$ defendiéndola de forma oral ante la autoridad judicial. La decisión de internar o no es adoptada por el/la juez/a de instrucción del lugar en el que se practicó la detención de la persona extranjera. Se trata de una decisión motivada que debe tomarse tras la celebración de una comparecencia en el juzgado, en la que participan la persona afectada y su abogado/a, así como el fiscal. La normativa vigente establece que la decisión judicial debe tener en cuenta las circunstancias concretas del caso, el principio de proporcionalidad $\mathrm{y}$, en especial, el riesgo de incomparecencia de la persona extranjera, sus actuaciones tendentes a dificultar la expulsión, así como la existencia de condenas o sanciones previas pendientes.

La aplicación de todos estos requisitos suscita muchos problemas en la práctica que tienen que ver con la indeterminación de dichos requisitos, así como los medios materiales y personales que existen durante todo el proceso. Pero en términos comparados, la regulación española es garantista y permite atribuir los internamientos que vulneran la regulación vigente a errores en cadena.

Los datos oficiales existentes sobre la práctica del internamiento en el conjunto del Estado solo hacen referencia a las solicitudes policiales y el sentido de los informes del Ministerio Fiscal. Como se desprende de la Tabla 1, en el período 2011-2015 el número de solicitudes policiales de internamiento se redujo en un $37,4 \%$, pasando de 14.004 a 8.765. En contraste con esta tendencia, cabe destacar que el Ministerio Fiscal se opuso en el 25\% de las solicitudes presentadas como media.

Nada se sabe, en cambio, de las decisiones judiciales que autorizaron o denegaron las solicitudes policiales de internamiento. Las Memorias de la Fiscalía General del Estado no recogen datos al respecto. Los Informes del Defensor del Pueblo como Mecanismo Nacional de Prevención de la Tortura ofrecen cifras sobre el número de extranjeros internados en los distintos $\mathrm{CIE}^{9}$, pero no sobre el resultado final de los expedientes de internamiento.

9 De acuerdo con estos informes (consultables en www.defensordelpueblo. es), en el año 2011 se internaron un total de 11.456 personas extranjeras. Esta cifra se redujo a 11.325 en el año 2012, a 9.002 en 2013, a 7.349 en 2014 y a 6.930 en 2015 , lo cual supuso una disminución total del $40 \%$. 
TABLA 1

SOLICITUDES POLICIALES DE INTERNAMIENTO Y POSICIÓN DEL MINISTERIO FISCAL EN EL CONJUNTO DEL ESTADO

\begin{tabular}{|l|r|r|r|r|r|}
\hline & 2011 & 2012 & 2013 & 2014 & 2015 \\
\hline $\begin{array}{l}\text { Solicitudes policiales } \\
\text { de internamiento }\end{array}$ & 14.004 & 11.760 & 10.063 & 8.671 & 8.765 \\
\hline $\begin{array}{l}\text { Informes favorables del } \\
\text { Ministerio Fiscal }\end{array}$ & $10.272(73,3 \%)$ & $8.807(74,9 \%)$ & $6.988(69,4 \%)$ & $\begin{array}{r}6.761 \\
(78 \%)\end{array}$ & $7.503(80 \%)$ \\
\hline $\begin{array}{l}\text { Informes desfavorables } \\
\text { del Ministerio Fiscal }\end{array}$ & $3.732(26,7 \%)$ & $2.953(25,1 \%)$ & $3.075(30,6 \%)$ & $\begin{array}{r}1.860 \\
(22 \%)\end{array}$ & $1.262(20 \%)$ \\
\hline
\end{tabular}

Fuente: Memorias de la Fiscalía General del Estado.

\section{DATOS Y METODOLOGÍA}

El presente trabajo pretende dar respuesta a las preguntas formuladas anteriormente a partir del análisis de los expedientes de internamiento tramitados en la provincia de Barcelona en el año $2015^{10}$. La elección de esta provincia obedece a diversos motivos: por un lado, a la inestimable colaboración prestada por la Fiscalía de Barcelona al permitir el acceso a todos los expedientes tramitados en la provincia ${ }^{11}$; por otro lado, a que se trata de una provincia que cuenta con un número importante de personas extranjeras procedentes de muchos países ${ }^{12}$; y por último, a que por razones geográficas la práctica del internamiento en Barcelona no está dominada por la devolución, es decir, por el intento de repatriar

10 La investigación ha podido realizarse gracias a la financiación recibida del proyecto de Barak Kalir The Social Life of State Deportation Regimes: A Comparative Study of the Implementation Interface, financiado por el European Research Council (ERC-Starting grant 336319).

11 En ejercicio de sus funciones de coordinación, la Fiscalía Delegada de Extranjería guarda copia de todos los expedientes tramitados en la provincia. Queremos dejar constancia de nuestra gratitud a Fernando Rodríguez Rey, Fiscal Delegado de Extranjería de Barcelona en el momento de su elaboración del estudio, por la ayuda prestada.

12 En el año 2011 había un total de 793.720 extranjeros empadronados en la provincia de Barcelona, cifra que descendió a 685.843 en el año 2015. No existen concentraciones significativas de personas de una determinada nacionalidad. Datos de Padrón continuo del Instituto de Estadística de Catalunya. 
a personas llegadas en patera o que han saltado la valla de Ceuta y Melilla, sino por la expulsión de personas que han permanecido antes en España durante varios años ${ }^{13}$.

En abril de 2016 accedimos a los expedientes. Un expediente típico cuenta con distintas piezas de información relevantes para el estudio: la solicitud policial de internamiento; el decreto de expulsión cuya ejecución se pretende garantizar; el informe del Ministerio Fiscal; el acta de la comparecencia del extranjero ante el juez; y el auto judicial autorizando o denegando el internamiento solicitado.

Con el objetivo de transformar la información contenida en los expedientes en datos analizables de manera sistemática, se construyó una base de datos específica. Tres estudiantes que recibieron formación para cumplimentar la base codificaron la información relevante de cada expediente en forma de variables.

La unidad de análisis en este estudio es un expediente que corresponde a un único individuo. Los datos tienen una estructura jerárquica y están anidados en juzgados de instrucción dentro de partidos judiciales. En la provincia de Barcelona lo habitual es que haya varios juzgados dentro de un mismo partido. Cada juzgado de instrucción corresponde a un único juez, si bien un mismo fiscal puede atender a más de un juzgado. La asignación del análisis de la solicitud entre los diversos juzgados de instrucción responde a reglas objetivas de reparto, puesto que las solicitudes se presenten al juzgado de guardia.

Por lo que se refiere a las variables de interés, los expedientes contienen información relevante sobre las características personales de las personas extranjeras (año de nacimiento, nacionalidad y sexo), así como de las circunstancias que rodearon la detención: dónde tuvo lugar y qué cuerpo policial la realizó.

Por lo que respecta a la motivación de las solicitudes policiales de internamiento y de las decisiones de fiscales y jueces, la interfaz de la base de datos utilizada por el equipo de codificación contenía un número de argumentos potenciales, deducidos de una primera revisión cualitativa de una muestra de expedientes, así como de los criterios legales para motivar el internamiento. La lista incluye,

13 Como se desprende de las Memorias de la Fiscalía de Barcelona, un número variable de personas internadas en el CIE de Barcelona sí que tienen órdenes de devolución por haber entrado irregularmente en España. Pero su internamiento no es decretado por jueces de Barcelona, sino por jueces radicados en los lugares donde se producen estas entradas. 
entre otros, los siguientes descriptores: existencia de antecedentes policiales, existencia de antecedentes penales, carencia de domicilio fijo y estable, carencia de medios económicos, falta de arraigo familiar, uso de nombres supuestos, documentación en trámite y necesidad de recabar información por parte de otros juzgados.

Además de analizar los expedientes de internamiento, nuestro estudio también ha tenido en cuenta las entrevistas realizadas al Fiscal Delegado de Extranjería de Barcelona, así como al Director del Centro de Internamiento de Barcelona y algunos miebros de la Brigada Provincial de Extranjería, que nos han aportado valiosa información sobre la práctica del internamiento.

En la sección siguiente se presentan los principales resultados de la investigación mediante tablas de frecuencias cruzadas.

\section{LOS PRINCIPALES HALLAZGOS DEL ESTUDIO}

En el año analizado (2015) se tramitaron en Barcelona 575 expedientes de internamiento. El importante descenso respecto del año anterior (en el que se tramitaron 911) se debió sobre todo a que el Centro de Internamiento de Extranjeros de Barcelona permaneció cerrado por obras desde el mes de octubre hasta el final del año ${ }^{14}$. La evolución decreciente del número de solicitudes de internamiento se inició, sin embargo, mucho antes y ha sido más acusada en Barcelona que en el conjunto del Estado. Así, como se desprende de las Tablas 1 y 2, en el período 2011-2015 en la provincia de Barcelona las solicitudes de internamiento descendieron el 62,51\%, mientras que en el conjunto del Estado lo hicieron el 37,4\%. Por su parte, en esta provincia los informes contrarios a dicha solicitud por parte del Ministerio Fiscal se situaron de promedio en el $28,8 \%$, esto es, 3 puntos por encima de la media estatal.

Lo más destacado, sin embargo, de la provincia catalana es el dato relativo a las decisiones judiciales sobre internamiento. A diferencia de la Memoria de la Fiscalía General del Estado, las

14 Como reconocieron los responsables policiales entrevistados durante la elaboración del trabajo, el coste y los problemas derivados del traslado de las personas extranjeras a otros centros de internamiento redundó en una reducción muy significativa de las solicitudes de internamiento durante los meses en que el CIE de Barcelona no estuvo operativo. 
Memorias anuales de la Fiscalía de Barcelona sí ofrecen datos al respecto. Así, como puede apreciarse en la Tabla 2, en el año 2015 los jueces de instrucción de Barcelona rechazaron algo más de la mitad de las solicitudes policiales. Este elevado número de denegaciones también se produjo en años anteriores. Pero lo que llama la atención es que, en un contexto de fuerte reducción del número de solicitudes de internamiento, los jueces de Barcelona siguen considerando que la mitad de las solicitudes policiales no estaban bien motivadas ${ }^{15}$.

TABLA 2

EXPEDIENTES DE INTERNAMIENTO TRAMITADOS EN LA PROVINCIA DE BARCELONA

\begin{tabular}{|l|r|r|r|r|r|}
\hline & 2011 & 2012 & 2013 & 2014 & \multicolumn{1}{c|}{2015} \\
\hline $\begin{array}{l}\text { Solicitudes policiales de } \\
\text { internamiento }\end{array}$ & 1.534 & 1.383 & 1.256 & 911 & 575 \\
\hline $\begin{array}{l}\text { Informes favorables del } \\
\text { Ministerio Fiscal }\end{array}$ & $1.227(80 \%)$ & $\begin{array}{r}925 \\
(66,9 \%)\end{array}$ & $850(67,7 \%)$ & $\begin{array}{r}598 \\
(65,6 \%)\end{array}$ & $383(66,6 \%)$ \\
\hline $\begin{array}{l}\text { Informes desfavorables } \\
\text { del Ministerio Fiscal }\end{array}$ & 307 & $458(33,1 \%)$ & $406(32,3 \%)$ & $\begin{array}{r}313 \\
(34,3 \%)\end{array}$ & $192(33,4 \%)$ \\
\hline $\begin{array}{l}\text { Autorizaciones judiciales } \\
\text { de internamiento }\end{array}$ & $724(47,2 \%)$ & $712(51,5 \%)$ & $625(49,8 \%)$ & $\begin{array}{r}432 \\
(47,4 \%)\end{array}$ & $\begin{array}{r}284 \\
(49,3 \%)\end{array}$ \\
\hline $\begin{array}{l}\text { Denegaciones judiciales } \\
\text { de internamiento }\end{array}$ & $810(52,8 \%)$ & $671(48 \%)$ & $631(50,2 \%)$ & $\begin{array}{r}479 \\
(52,6 \%)\end{array}$ & $\begin{array}{r}291 \\
(50,6 \%)\end{array}$ \\
\hline
\end{tabular}

Fuente: Memorias anuales de la Fiscalía Delegada de Extranjería de la provincia de Barcelona.

\subsection{El perfil de los extranjeros afectados por solicitudes de internamiento}

El análisis de los expedientes de internamiento también ha permitido poner de relieve algunas cuestiones interesantes en relación con las personas extranjeras cuyo internamiento fue solicitado por la policía en el año 2015. El primer dato significativo es que la inmensa mayoría de solicitudes incoadas en la provincia de Barcelona (96\%) afectó a varones. Esta importantísima desviación por sexo tiene una explicación clara: el CIE de Barcelona carece de un módulo para mujeres, de modo

15 Como se desprende de la Memoria de la Fiscalía de Barcelona, esta tendencia ha aumentado de manera significativa en el año 2016, en el que el 74\% de las solicitudes de internamiento fueron rechazadas. 
que toda mujer con una orden de internamiento expedida en Barcelona debe ser trasladada a los CIE de Valencia o Madrid, que sí cuentan con módulo para mujeres. Los datos muestran que los únicos expedientes correspondientes a mujeres se refieren a prohibiciones de entrada que tienen lugar en el aeropuerto de El Prat. Una cuestión meramente organizativa como la existencia o no de un módulo de mujeres en el CIE determina, pues, que una medida de privación de libertad se aplique en la práctica de forma tan desigual en función del sexo.

\section{TABLA 3}

\section{EXPEDIENTES DE INTERNAMIENTO POR NACIONALIDAD (PAÍSES CON CIFRAS SUPERIORES AL 2\%)}

\begin{tabular}{|l|c|}
\hline Nacionalidad & Porcentaje de solicitudes (N) \\
\hline Marruecos & $39,16(224)$ \\
\hline Ecuador & $5,07(29)$ \\
\hline Gambia & $4,3(25)$ \\
\hline Albania & $4,02(23)$ \\
\hline República Dominicana & $4,02(23)$ \\
\hline Colombia & $3,85(22)$ \\
\hline Georgia & $3,67(21)$ \\
\hline Bolivia & $3,5(20)$ \\
\hline Senegal & $3,5(20)$ \\
\hline Chile & $2,8(16)$ \\
\hline Perú & $2,8(16)$ \\
\hline Argelia & $2,45(14)$ \\
\hline Honduras & $2,27(13)$ \\
\hline Pakistán & $2,27(13)$ \\
\hline
\end{tabular}

Fuente: elaboración propia.

La investigación también ha puesto de manifiesto que los expedientes tramitados afectaron a personas de 47 nacionalidades diferentes ${ }^{16}$. Pero como se desprende de la Tabla 3, las solicitudes

16 A pesar de las dudas que existen en relación con la legalidad del internamiento de ciudadanos comunitarios, el 1,56\% de los expedientes incoados afectó a ciudadanos europeos. En concreto, 7 expedientes se tramitaron respecto de ciudadanos rumanos (el $1,22 \%$ del total) y 1 respecto de un ciudadano portugués y de otro lituano $(0,17 \%$ en cada caso) 
de internamiento no tuvieron el mismo impacto en todas las nacionalidades, sino que afectaron de forma muy destacada (casi el $40 \%$ ) a ciudadanos marroquíes.

Como han señalado otros estudios ${ }^{17}$, el internamiento no afecta por igual a las personas de las diversas nacionalidades. Pero esta circunstancia no se da solo en el interior de los CIE, sino en las propias solicitudes policiales de internamiento. El contraste de los expedientes analizados con el número de personas de las diferentes nacionalidades empadronadas en la provincia de Barcelona en el año 2015 pone de relieve, en efecto, que algunas nacionalidades están claramente infrarrepresentadas en las solicitudes policiales, mientras que otras están sobrerrepresentadas. Así, como se refleja en el Gráfico 1, la marroquí es la nacionalidad más sobrerrepresentada, a pesar de que la colonia marroquí también es la más numerosa en esta provincia. En el otro extremo, los nacionales de Argentina, Ecuador o China tuvieron porcentajes de solicitudes de internamiento muy inferiores al número de ciudadanos de estos países empadronados en la provincia de Barcelona.

Las causas de este hallazgo son seguramente múltiples, pero ponen de relieve que las solicitudes de internamiento no son neutras desde el punto de vista de la nacionalidad, sino que tienen en cuenta diversos factores: en unos casos, la viabilidad de las expulsiones (existencia de acuerdos de readmisión con los países de origen y colaboración de las autoridades para documentar a sus nacionales) juega un papel relevante tanto en sentido positivo como negativo ${ }^{18}$; en otros casos, la existencia de colectivos de algunas nacionalidades relacionados con determinados fenómenos delictivos parece contribuye a priorizar su expulsión y su internamiento como una política de seguridad pública ${ }^{19}$. En cambio, la mayor o menor representatividad de determinadas nacionalidades no parece influida por criterios étnicos o geográficos.

17 García España, E. (2017), Martínez Escamilla, M. (2015) y Solanes Corella, A. (2016), entre otros.

18 Marruecos y Argelia serían ejemplos en el primer sentido, mientras que la infrarrepresentación de personas rusas o ecuatorianas parecen influidas, según las entrevistas realizadas a los funcionarios policiales durante la elaboración de este trabajo, por la dificultad de documentar a estas personas para su expulsión.

19 Este sería el caso, según los mismos testimonios policiales, de los ciudadanos de Albania, Georgia y Gambia. 
GRÁFICO 1

\section{REPRESENTACIÓN RELATIVA DE NACIONALIDADES EN LAS SOLICITUDES DE INTERNAMIENTO}
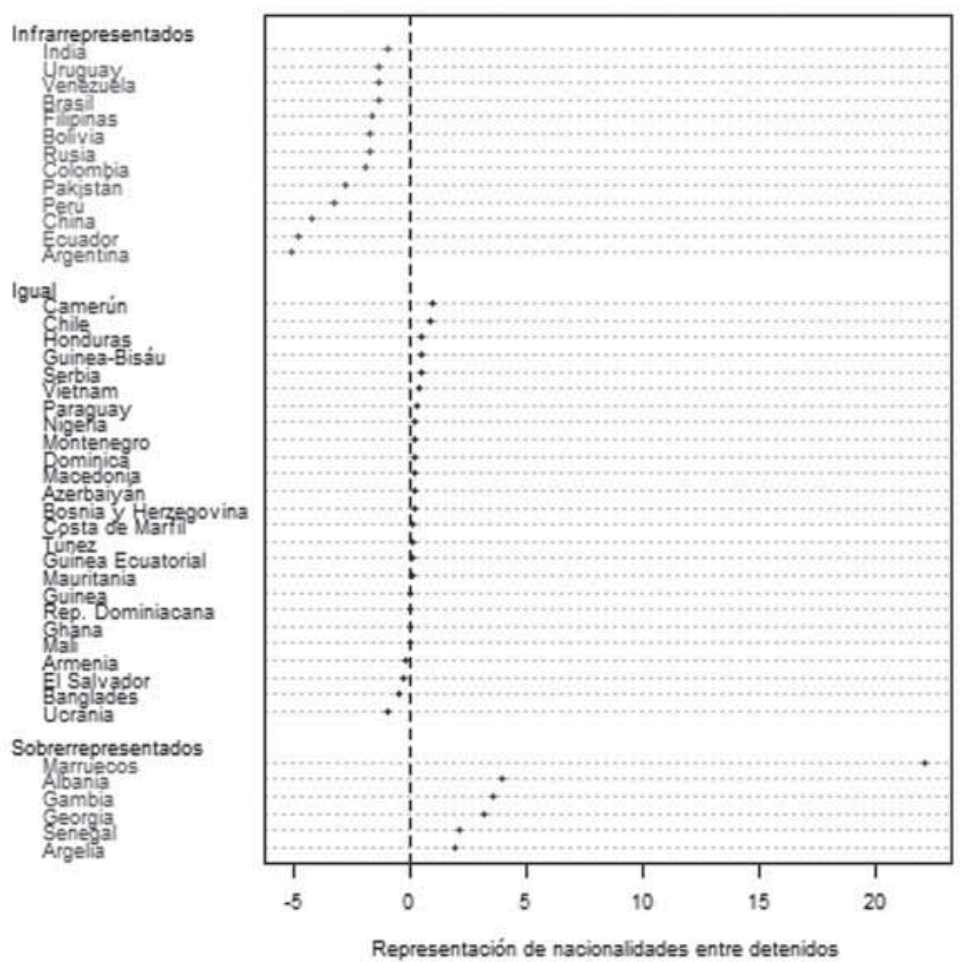

Fuente: elaboración propia.

\subsection{Las solicitudes policiales de internamiento}

Otra de las cuestiones analizadas en el estudio ha sido las solicitudes de internamiento incoadas por funcionarios del Cuerpo Nacional de Policía en la provincia de Barcelona. La Circular 6/2014 de la Dirección General de la Policía sobre criterios para solicitar el ingreso de ciudadanos extranjeros en los centros de internamiento obliga a los instructores de estos procedimientos a valorar, con carácter previo a la solicitud de internamiento, una serie de circuns- 
tancias personales del extranjero, así como la posibilidad real de ejecutar la repatriación ${ }^{20}$.

Como se desprende de la Tabla 4, la Unidad Central de Redes de Inmigración Ilegal y Falsedades Documentales (UCRIF) de Barcelona fue la que incoó el mayor número de expedientes $(35,8 \%)$ en el período analizado. Ello es lógico, si se tiene en cuenta que en el caso de Barcelona esta Unidad centraliza todos los expedientes iniciados en la ciudad de Barcelona y los que tienen su origen en investigaciones de los diversos grupos operativos de dicha Unidad. Las restantes solicitudes de internamiento (el 64,2\%) fueron presentadas por comisarías locales (CL) del Cuerpo Nacional de Policía ubicadas en la provincia de Barcelona, que no son muy numerosas al ser los Mossos d'Esquadra la policía integral de Cataluña.

El diverso protagonismo de las distintas comisarías obedece a su ámbito territorial de actuación y, sobre todo, a su proximidad a puestos fronterizos o centros penitenciarios. El primero es el caso de la Comisaría del Aeropuerto, que tramita el internamiento de todas las personas con órdenes de expulsión detectadas en el aeropuerto y de aquellas que son rechazadas en frontera y no permanecen en la sala de inadmitidos. El segundo es el caso de las comisarías de Sabadell, Terrassa y Manresa, que están próximas a los centros penitenciarios de Brians y Lledoners, y que solicitan el internamiento de las personas que se pretende expulsar por haber

20 En el caso de las circunstancias de carácter personal, la Circular distingue, por un lado, las genéricas (la existencia de domicilio conocido, la convivencia y vínculos familiares, la existencia de hijos menores a cargo, las consecuencias de la expulsión para el extranjero y su familia, el arraigo en España, estar provisto de pasaporte, el riesgo de fuga o de incomparecencia, representar un riesgo para el orden público o la seguridad pública o nacional o cualquier otra circunstancia relativa a la existencia de vínculos con España o con su país de origen) y, por otro, las circunstancias específicas (edad, estar embarazada, estado físico y psíquico, necesidad de tratamiento médico, haber padecido tortura o algún tipo de violencia física, psíquica, sexual o de cualquier otro tipo, o ser una persona vulnerable). Por lo que se refiere a las circunstancias sobre la posibilidad real de ejecutar la repatriación, la Circular hace referencia a hechos como estar provisto de documento de viaje o pasaporte, la existencia de representación consular en España, la posibilidad de ser documentado por las autoridades de su país y el que la repatriación sea factible en el plazo máximo de 60 días. El cumplimiento real de estos requisitos es imposible de comprobar, pero queda en entredicho en los supuestos en que se internan a personas en relación con las cuales dichos requisitos no se dan de forma clara. 
sido condenadas por delito doloso a penas superiores a un año (art. 57.2 de la Ley de Extranjería).

\section{TABLA 4}

UNIDADES POLICIALES QUE HAN INCOADO LOS EXPEDIENTES DE INTERNAMIENTO

\begin{tabular}{|l|c|}
\hline Unidad policial CNP & \% Solicitudes de internamiento \\
\hline UCRIF-Barcelona & 35,8 \\
\hline CL Sabadell & 11,46 \\
\hline CL Terrassa & 11,11 \\
\hline Aeropuerto de Barcelona & 10,41 \\
\hline CL Cornellà-Esplugues de Llobregat & 7,76 \\
\hline CL Manresa & 7,76 \\
\hline Media & 7,69 \\
\hline CL Mataró & 6,17 \\
\hline CL L'Hospitalet de Llobregat & 4,41 \\
\hline CL Sant Adrià de Besòs & 2,65 \\
\hline CL Vilanova i la Geltrú & 1,23 \\
\hline CL Vic & 0,53 \\
\hline CL Granollers & 0,35 \\
\hline CL lgualada & 0,35 \\
\hline
\end{tabular}

Fuente: elaboración propia.

La investigación también ha revelado que las detenciones que desencadenaron la solicitud de internamiento fueron practicadas en la mitad de los supuestos por el Cuerpo Nacional de Policía, en el $33 \%$ de los casos por la policía autonómica y en el $17 \%$ por las diversas policías locales. En la medida en que las competencias de control de inmigración son exclusivas del Cuerpo Nacional de Policía, cabe concluir que la mayor parte de las detenciones estuvo 
relacionada, pues, con el ejercicio de las competencias en materia de seguridad ciudadana y no con controles de inmigración.

Casi el $60 \%$ de las detenciones tuvo lugar en la calle, mientras que el $15 \%$ se produjo en el aeropuerto. Pero lo más llamativo es que el 25\% de las detenciones se practicó en las inmediaciones de centros penitenciarios, lo cual no parece casual, sino que obedece a la decisión política de priorizar la expulsión de personas en las que concurre el supuesto del art. 57.2 de la Ley de Extranjería, esto es, haber sido condenadas por una conducta dolosa constitutiva de delito a una pena privativa de libertad de más de un año. Como se verá a continuación, esta infracción estuvo en el origen de más de un tercio de las órdenes de expulsión que se pretendieron garantizar mediante las solicitudes de internamiento incoadas. En todo caso, el importante número de detenciones realizadas en las inmediaciones de los centros penitenciarios revela la existencia de un importante nivel de cooperación entre los responsables de los centros penitenciarios (que en Cataluña son competencia del Gobierno autonómico) y los diversos cuerpos policiales, cuyos agentes están esperando al extranjero a la salida de prisión para proceder a su detención y a la incoación de los correspondientes expedientes de expulsión y de internamiento.

Por lo que se refiere a la orden de salida obligatoria cuya ejecución se pretende garantizar, la investigación ha puesto de relieve que la inmensa mayoría $(90 \%)$ de las solicitudes de internamiento analizadas estuvieron relacionadas con órdenes de expulsión. El $86 \%$ de estas órdenes habían sido dictadas por la Subdelegación del Gobierno de Barcelona. La mayor parte de estas expulsiones (el 66,47\%) sancionaron supuestos de estancia irregular, mientras que el $31,72 \%$ se basó en la previa existencia de una condena penal superior a 1 año.

En casi todos los supuestos el expediente de internamiento se incoó una vez decretada la orden de expulsión y no durante la tramitación del expediente de expulsión. De hecho, la investigación también ha puesto de relieve que la media de días transcurridos entre la orden de expulsión y la solicitud de internamiento fue elevada (484,7 días, es decir casi 1 año y 4 meses), si bien la desviación es alta (118,8 días). Por lo que respecta a la notificación de la orden de expulsión, el estudio ha puesto de manifiesto que en el 49,09\% de los casos se había realizado de manera personal y en 8,54\% de los supuestos a través de la asistencia letrada de la persona extranjera. 
En el restante $42,38 \%$ de los casos la notificación de la expulsión se había practicado a través de edictos, lo cual es muy relevante, puesto que es probable que en muchos de estos casos la persona extranjera no conociese realmente la existencia de dicha orden ${ }^{21}$.

\subsection{La motivación de las solicitudes policiales de internamiento}

El análisis de las solicitudes policiales de internamiento realizadas en la provincia de Barcelona en el año 2015 también ha permitido poner de relieve algunas cuestiones interesantes en relación con su motivación.

Por un lado, se ha constatado el uso de diversas plantillas o modelos de solicitud por parte de las distintas unidades policiales. Las diferencias formales no son radicales, pero este hecho revela las dificultades para que exista una unidad de criterio a pesar de la ya mencionada Circular del Cuerpo Nacional de Policía. Además, debe tenerse en cuenta que dichos modelos deben ser cumplimentados en muchos casos en poco tiempo ${ }^{22}$ y con los medios limitados que están al alcance del instructor ${ }^{23}$. Todo ello redunda en que la motivación de las solicitudes de internamiento sea casi siempre breve, no se acompañe ni de información detallada sobre los motivos aducidos, ni de una valoración de conjunto de los motivos que justifican, según la policía, la necesidad del internamiento.

Por otro lado, como se desprende de la Tabla 5, los motivos aducidos para solicitar el internamiento fueron varios: las solicitudes policiales se basaron sobre todo en la existencia de antecedentes

21 El estudio descarta que esta circunstancia tuviese incidencia en la valoración del riesgo de incomparecencia por parte de los jueces de instrucción. Así, en el 58,39\% de los casos la autorización judicial fue denegada en supuestos de órdenes de expulsión notificadas por edictos, mientras que dicho porcentaje alcanzó el 55,26\% y el 51,85\% en los supuestos en que las órdenes de expulsión se notificaron de forma personal o a través de abogado.

22 Siempre dentro de las 72 horas desde la detención del extranjero. Piénsese, sin embargo, que en la mitad de los casos dicha detención fue practicada por un cuerpo policial distinto, de modo que los funcionarios del Cuerpo Nacional de Policía dispusieron de bastante menos tiempo para elevar la petición de internamiento.

23 En este sentido, debe tenerse en cuenta que el instructor tiene acceso a las diversas bases de datos policiales, pero no así al Registro de Condenados y Penados, donde pueden consultarse los antecedentes penales del extranjero. 
policiales (en $96 \%$ de los casos) ${ }^{24}$ y, a bastante distancia, en la existencia de antecedentes penales (52\%), en la carencia de domicilio fijo y estable (49\%), en la carencia de medios económicos (41\%), en la falta de arraigo familiar (38\%), en el uso de nombres supuestos $(38 \%)$ y en otros argumentos.

TABLA 5

ARGUMENTOS ADUCIDOS POR LA POLICÍA PARA SOLICITAR EL INTERNAMIENTO

\begin{tabular}{|l|c|}
\hline Fundamento del internamiento & Expedientes en que se aducen (\%) \\
\hline Existencia de antecedentes policiales & 96,42 \\
\hline Existencia de antecedentes penales & 52,46 \\
\hline Carencia de domicilio fijo y estable & 49,60 \\
\hline Carencia de medios económicos & 40,98 \\
\hline Falta de arraigo familiar & 38,46 \\
\hline Uso de nombres supuestos & 38,46 \\
\hline Documentación en trámite & 5,52 \\
\hline Necesidad de recabar documentación de otros Juzgados & 4,74 \\
\hline Otros argumentos & 84,32 \\
\hline
\end{tabular}

Fuente: elaboración propia.

En la práctica, resulta claro que las solicitudes de internamiento están muy influidas por consideraciones de seguridad. Así, el peso otorgado a los antecedentes policiales y penales (que se corresponde con el criterio político de priorizar las llamadas "expulsiones preferentes o cualificadas") y el criterio de la carencia de medios económicos parecen más relacionados con la supuesta "peligrosidad" del extranjero que con la necesidad de garantizar su expulsión mediante la privación de libertad. Teniendo en cuenta los problemas que sus-

24 Los porcentajes de la Tabla 6 no suman 100 porque una misma solicitud puede contener más de un fundamento. 
citan todos estos conceptos ${ }^{25}$, el estudio ha profundizado en el uso práctico de los antecedentes policiales, de los antecedentes penales y de la carencia de domicilio fijo y estable.

Por lo que respecta a los antecedentes policiales, la investigación ha puesto de relieve no solo su invocación generalizada, sino que el número de los antecedentes que tenían las personas cuyo internamiento se solicitó era elevado (9,44 de media). La heterogeneidad de estos antecedentes también fue grande (58 tipos distintos, expresados a través de conceptos sobre todo penales), aunque los más citados en primer lugar fueron la reclamación judicial, las infracciones de la Ley de extranjería, el robo con fuerza, el robo con violencia y los malos tratos ${ }^{26}$.

El propio concepto de antecedente policial plantea problemas jurídicos importantes derivados de su indefinición y falta de regulación. En el caso concreto del internamiento, cabe recordar que los antecedentes policiales no aparecen entre los criterios de la Ley de Extranjería que fundamentan el internamiento, aunque estos sí contemplan la existencia de sanciones administrativas previas y de procedimientos sancionadores pendientes. Pero como pone de relieve la invocación de las infracciones de la Ley de extranjería, el concepto de antecedente que se emplea en la práctica no siempre se reserva a conductas con relevancia penal. A su vez, la mera cita del antecedente sin ninguna información adicional pone de relieve que el concepto de antecedente policial manejado en las solicitudes de internamiento analizadas aparece vinculado a una idea amplia de peligrosidad que puede ser legítima desde un punto de vista policial. Pero no no debería trasladarse sin más a la solicitud de internamiento, a no ser que el concreto antecedente esté directamente vinculado con el riesgo de incomparecencia a efectos de expulsión. El estudio realizado corrobora la necesidad de hacer un uso más cuidadoso de los antecedentes policiales que, entre otras cosas, tenga en cuenta el tipo de antecedente en que se ha incurrido y que no parta de la base

25 Algunos autores han criticado especialmente el concepto de antecedente policial, que no tiene una definición legal y que suele englobar detenciones policiales por cualquier motivo y con independencia de si se produjo una puesta a disposición judicial o no. Por todos, vid. Martínez Escamilla, M. (2016). En relación con el uso de los antecedentes penales en relación con algunas figuras de extranjería, vid. Larrauri, E. (2016).

26 Aunque con otro orden, estos también fueron los antecedentes más frecuentes mencionados en segundo, tercero, cuarto y quinto lugar. 
que cualquier antecedente es un indicio de que el extranjero no podrá ser localizado en el momento de ejecutar su expulsión ${ }^{27}$.

Por lo que respecta a los antecedentes penales, el estudio también ha puesto de relieve que juegan un papel muy importante en las solicitudes de internamiento. La existencia de este tipo de antecedentes aparece en la mitad $(52,6 \%)$ de los expedientes, aunque su número fue mucho más bajo que el de los antecedentes policiales $(1,6$ como media frente a 9,44). Asimismo, cabe señalar que la heterogeneidad de los antecedentes penales fue elevada (36 tipos distintos), aunque inferior a la de los antecedentes policiales (58). Los cinco antecedentes penales más invocados fueron, por este orden, el robo con violencia (19,51\% de los expedientes), los delitos contra la salud pública $(17,89 \%)$, los delitos de lesiones y de robo con fuerza en las cosas (en ambos casos, en el 5,69\%), el delito de agresión sexual $(4,88 \%)$ y el de hurto $(4,07 \%)$.

Como en el caso de los antecedentes policiales, la referencia a este tipo de antecedentes se limita a una mera cita, sin que conste si se había consultado el Registro Central de Penados, que, por otro lado, solo es accesible para algunos funcionarios policiales. Ciertamente, la existencia de condenas penales previas constituye uno de los criterios legales que deben ser empleados en la autorización judicial del internamiento. Pero la investigación ha puesto de relieve que las solicitudes de internamiento se limitan a citar su existencia, sin aportar datos sobre la situación concreta de dichos antecedentes ni sobre la proporcionalidad de la medida de internamiento a la luz de los mismos.

La mera invocación de su concurrencia también es una constante en los demás motivos aducidos por la policía para justificar la solicitud de internamiento. En el caso concreto de la carencia de domicilio fijo y estable, el estudio revela que en el $70 \%$ de los casos su concurrencia se basó en los datos del padrón municipal de habitantes, es decir, en la constancia de cambios frecuentes de empadronamiento. No se refleja la realización de comprobaciones policiales sobre la situación de hecho en el momento de solicitar el internamiento, lo cual permitiría excluir los supuestos en que dichos cambios están vinculados a la situación económica de la persona, sin que ello se traduzca en una alta probabilidad de incomparecencia en el momento de ejecutar la expulsión.

27 Así, una incomparecencia contrastada a una citación judicial podría tener, a efectos de internamiento, un peso mayor que un antecedente por otro delito. 


\subsection{El papel del Ministerio Fiscal en los expedientes de internamiento}

Como se ha señalado, el Ministerio Público participa en los expedientes de internamiento haciendo un control de legalidad de la solicitud policial. La regulación de su intervención es precaria y ha sido completada por sucesivas Circulares de la Fiscalía General del Estado. La actualmente vigente (la 2/2006, sobre diversos aspectos relativos al régimen de los extranjeros en España) señala la conveniencia que dicha participación se produzca de dos modos: mediante la emisión de informe previo a la decisión judicial y mediante la presencia del fiscal en la comparecencia del extranjero ante el juez de instrucción. La intervención del fiscal debe inspirarse en su función de patronus libertatis y debe tener en cuenta la excepcionalidad del internamiento como medida cautelar.

La investigación desarrollada ha puesto de relieve, sin embargo, que la presencia del Ministerio Fiscal en la comparecencia del extranjero solo queda acreditada en el 59\% de los casos. El criterio del fiscal está presente, ya sea por escrito, ya sea de forma oral, en todos los expedientes de internamiento. Pero la forma de aportarlo parece influida en la práctica por la plantilla de fiscales que existe en los diversos juzgados de instrucción.

Por lo que respecta al criterio del Ministerio Fiscal en relación con la solicitud policial de internamiento, el estudio pone de relieve que en el $33,4 \%$ de los casos fue contrario a esta medida y en el $66,6 \%$ favorable. Estos porcentajes son similares a de los años anteriores, lo cual no deja de ser llamativo en un contexto de reducción del número de solicitudes de internamiento. También cabe destacar que se han constatado diferencias notables entre los fiscales adscritos a los distintos juzgados de instrucción. Así, como se desprende de la Tabla 6, el porcentaje de oposición a las solicitudes de internamiento se situó entre el 0 y el $71 \%$ en aquellos juzgados que tramitaron más de 5 solicitudes. Estas diferencias pueden deberse a las características de los diversos expedientes. Sin embargo, llama la atención que también se den entre juzgados de una misma localidad y que en 11 juzgados el informe favorable del Ministerio Fiscal se haya producido en el $100 \%$ de los supuestos. 
TABLA 6

SENTIDO DEL INFORME DEL MINISTERIO FISCAL EN RELACIÓN CON LAS SOLICITUDES DE INTERNAMIENTO POR JUZGADOS. SE INCLUYEN SÓLO AQUELLOS JUZGADOS CON MÁS DE 5 EXPEDIENTES

\begin{tabular}{|c|c|c|c|c|c|}
\hline \multirow{2}{*}{ Juzgado de Instrucción } & \multirow{2}{*}{ Favorable } & \multirow{2}{*}{ Desfavorable } & \multirow{2}{*}{$N$} & \multicolumn{2}{|c|}{ Porcentaje } \\
\hline & & & & Favorable & Desfavorable \\
\hline Cerdanyola del Vallès 7 & 2 & 5 & 7 & 28.57 & 71.43 \\
\hline L'Hospitalet de Llobregat 1 & 2 & 5 & 7 & 28.57 & 71.43 \\
\hline Barcelona 15 & 3 & 3 & 6 & 50.00 & 50.00 \\
\hline Granollers 4 & 4 & 4 & 8 & 50.00 & 50.00 \\
\hline Sabadell 4 & 3 & 3 & 6 & 50.00 & 50.00 \\
\hline Terrassa 1 & 9 & 8 & 17 & 52.94 & 47.06 \\
\hline Sabadell 3 & 5 & 4 & 9 & 55.56 & 44.44 \\
\hline Barcelona 31 & 3 & 2 & 5 & 60.00 & 40.00 \\
\hline Barcelona 7 & 3 & 2 & 5 & 60.00 & 40.00 \\
\hline Granollers 1 & 3 & 2 & 5 & 60.00 & 40.00 \\
\hline Sabadell 2 & 3 & 2 & 5 & 60.00 & 40.00 \\
\hline Barcelona 29 & 5 & 3 & 8 & 62.50 & 37.50 \\
\hline Martorell 3 & 5 & 3 & 8 & 62.50 & 37.50 \\
\hline Granollers 3 & 6 & 3 & 9 & 66.67 & 33.33 \\
\hline Manresa 1 & 4 & 2 & 6 & 66.67 & 33.33 \\
\hline Terrassa 2 & 10 & 4 & 14 & 71.43 & 28.57 \\
\hline Martorell 6 & 6 & 2 & 8 & 75.00 & 25.00 \\
\hline Terrassa 3 & 9 & 3 & 12 & 75.00 & 25.00 \\
\hline Sabadell 5 & 7 & 2 & 9 & 77.78 & 22.22 \\
\hline Terrassa 4 & 7 & 2 & 9 & 77.78 & 22.22 \\
\hline Barcelona 28 & 4 & 1 & 5 & 80.00 & 20.00 \\
\hline Martorell 7 & 4 & 1 & 5 & 80.00 & 20.00 \\
\hline Martorell 5 & 10 & 2 & 12 & 83.33 & 16.67 \\
\hline Rubí 7 & 5 & 1 & 6 & 83.33 & 16.67 \\
\hline
\end{tabular}




\begin{tabular}{|c|c|c|c|c|c|}
\hline \multirow{2}{*}{ Juzgado de Instrucción } & \multirow{2}{*}{ Favorable } & \multirow{2}{*}{ Desfavorable } & \multirow{2}{*}{$N$} & \multicolumn{2}{|c|}{ Porcentaje } \\
\hline & & & & Favorable & Desfavorable \\
\hline El Prat de Llobregat 3 & 7 & 1 & 8 & 87.50 & 12.50 \\
\hline Manresa 5 & 7 & 1 & 8 & 87.50 & 12.50 \\
\hline Martorell 2 & 7 & 1 & 8 & 87.50 & 12.50 \\
\hline Martorell 4 & 8 & 1 & 9 & 88.89 & 11.11 \\
\hline Granollers 2 & 9 & 1 & 10 & 90.00 & 10.00 \\
\hline El Prat de Llobregat 5 & 15 & 1 & 16 & 93.75 & 6.25 \\
\hline Barcelona 1 & 5 & 0 & 5 & 100.00 & 0.00 \\
\hline Barcelona 26 & 6 & 0 & 6 & 100.00 & 0.00 \\
\hline El Prat de Llobregat 1 & 11 & 0 & 11 & 100.00 & 0.00 \\
\hline El Prat de Llobregat 2 & 10 & 0 & 10 & 100.00 & 0.00 \\
\hline El Prat de Llobregat 4 & 5 & 0 & 5 & 100.00 & 0.00 \\
\hline L'Hospitalet de Llobregat 3 & 7 & 0 & 7 & 100.00 & 0.00 \\
\hline L'Hospitalet de Llobregat 5 & 7 & 0 & 7 & 100.00 & 0.00 \\
\hline Manresa 4 & 8 & 0 & 8 & 100.00 & 0.00 \\
\hline Manresa 6 & 6 & 0 & 6 & 100.00 & 0.00 \\
\hline Manresa 7 & 6 & 0 & 6 & 100.00 & 0.00 \\
\hline Martorell 1 & 12 & 0 & 12 & 100.00 & 0.00 \\
\hline
\end{tabular}

Fuente: elaboración propia.

Por último, el estudio ha analizado los argumentos empleados por el Ministerio Fiscal para apoyar u oponerse a la solicitud policial de internamiento. El resultado más destacado de este análisis es que los argumentos del fiscal están sobre todo relacionados con el arraigo familiar, la estabilidad del domicilio y, en menor medida, los medios económicos de la persona en cuestión. Así, como se desprende de la Tabla 7, en el 57\% de los informes desfavorables se consideró que existía arraigo familiar y en el 53\% que el extranjero sí tenía domicilio estable. De igual modo, en el $61 \%$ de los informes favorables se adujo que el extranjero carecía de arraigo familiar, mientras que en el 36,4\% que carecía de medios económicos.

El estudio pone de relieve, pues, el cumplimiento general de la Circular de la Fiscalía General del Estado que regula el criterio de actuación de los fiscales en esta materia y que vincula el riesgo de 
incomparecencia con la socialización e integración de la persona extranjera y no con su posible peligrosidad derivada de la existencia de antecedentes policiales y penales. Es cierto que la intervención del fiscal es posterior a la de la policía y que los fiscales pueden acceder a alguna información (por ejemplo, a los antecedentes penales) de manera directa. Pero mientras la policía emplea criterios de seguridad, el Ministerio Fiscal acude sobre todo a consideraciones de integración para motivar su posición.

\section{TABLA 7}

MOTIVOS ADUCIDOS POR EL MINISTERIO FISCAL EN RELACIÓN CON LA SOLICITUD DE INTERNAMIENTO

\begin{tabular}{|l|c|}
\hline Argumento contrario al internamiento & Porcentaje de utilización \\
\hline Existencia de arraigo familiar & 57,6 \\
\hline Existencia de domicilio estable & 53,6 \\
\hline Existencia de arraigo social & 12 \\
\hline Existencia de medios económicos & 8,8 \\
\hline No firmeza de la expulsión & 7,2 \\
\hline Antecedentes policiales insuficientes & 4,8 \\
\hline Falta de notificación de la expulsión & 4,0 \\
\hline No obstaculización de la expulsión & 3,2 \\
\hline Existencia autorización de residencia & 1,6 \\
\hline Motivos de salud & 1,6 \\
\hline Otros & 22,4 \\
\hline Argumento favorable al internamiento & Porcentaje de utilización \\
\hline Carencia de arraigo familiar & 61,4 \\
\hline Carencia de medios económicos & 36,4 \\
\hline Carencia de domicilio estable & 25,7 \\
\hline Antecedentes penales & 14,3 \\
\hline Antecedentes policiales & 12,8 \\
\hline Otros & 25 \\
\hline & \\
\hline
\end{tabular}

Fuente: elaboración propia. 


\subsection{El papel de los abogados defensores de los extranjeros}

El estudio también ha analizado la actuación de los abogados durante la tramitación de los expedientes de internamiento. La legislación española contempla el derecho a la asistencia jurídica gratuita en todos los expedientes de internamiento, aunque en la práctica esta asistencia debe prestarse en la mayoría de supuestos con escasos márgenes de tiempo. Los expedientes analizados no permiten extraer mucha información sobre las condiciones en que se presta la asistencia letrada, puesto que lo único que consta es el acta de la comparecencia. Pero el estudio ha permitido obtener algunos datos relevantes sobre el papel de los letrados en la práctica.

Así, su presencia en la comparecencia se ha podido certificar en el 98\% de los casos, es decir, en casi todos los expedientes. Además, hay que tener en cuenta, como se ha visto, que en el $86 \%$ de los casos la orden de expulsión y el expediente de internamiento se habían tramitado en la provincia de Barcelona, lo cual debería facilitar el contacto entre el abogado del procedimiento de expulsión y el del expediente de internamiento. Por lo que respecta a los argumentos aducidos por los abogados en contra del internamiento los más frecuentes fueron la existencia de arraigo familiar (75\% de los supuestos), de domicilio conocido $(60 \%)$ y de medios económicos $(38 \%)$.

Pero lo que sin duda llama más la atención del análisis efectuado es que en casi un $20 \%$ de los casos el acta de la comparecencia ante el juez no refleja ningún tipo de intervención por parte del abogado. Al margen de las causas de este comportamiento, que pueden ser diversas, un comportamiento pasivo en la asistencia jurídica recibida por la persona afectada se compadece mal con la función atribuida a los abogados por la normativa vigente.

\subsection{El papel de los jueces de instrucción en la decisión sobre el internamiento}

Por último, el estudio ha analizado en detalle la actuación del juez que debe decidir sobre la solicitud de internamiento. Aunque no existe una regulación concreta de la audiencia que debe preceder a la decisión judicial, el estudio ha puesto de relieve que en la práctica suele celebrarse una vista en el propio Juzgado. De las actas 
de dicha comparecencia se desprende que las partes se limitan a exponer sus argumentos, que las comparecencias son breves y que son muy excepcionales las solicitudes de prueba.

Por lo que se refiere al contenido de la propia decisión judicial es muy significativo que en la mitad de los casos $(50,6 \%)$ los jueces de la provincia de Barcelona hayan rechazado las solicitudes de internamiento. En un contexto de disminución de las solicitudes de internamiento y de una actuación policial aparentemente más selectiva, el mantenimiento de una tasa de rechazo judicial del 50\% pone de manifiesto una importante oposición judicial a la institución del internamiento.

TABLA 8

DISTRIBUCIÓN DE DECISIONES JUDICIALES (AUTORIZACIÓN O DENEGACIÓN) POR JUZGADOS. SÓLO SE INCLUYEN JUZGADOS CON MÁS DE 10 EXPEDIENTES

\begin{tabular}{|c|c|c|c|c|c|}
\hline & & & & \multicolumn{2}{|c|}{ Porcentaje } \\
\hline & Autoriza & Deniega & $N$ & Autoriza & Deniega \\
\hline El Prat de Llobregat 1 & 12 & 0 & 12 & 100.00 & 0.00 \\
\hline El Prat de Llobregat 2 & 8 & 3 & 11 & 72.73 & 27.27 \\
\hline El Prat de Llobregat 3 & 8 & 5 & 13 & 61.54 & 38.46 \\
\hline El Prat de Llobregat 5 & 20 & 2 & 22 & 90.91 & 9.09 \\
\hline Granollers 2 & 3 & 7 & 10 & 30.00 & 70.00 \\
\hline Martorell 1 & 8 & 4 & 12 & 66.67 & 33.33 \\
\hline Martorell 4 & 1 & 9 & 10 & 10.00 & 90.00 \\
\hline Martorell 5 & 4 & 7 & 11 & 36.36 & 63.64 \\
\hline Terrassa 1 & 9 & 8 & 17 & 52.94 & 47.06 \\
\hline Terrassa 2 & 6 & 8 & 14 & 42.86 & 57.14 \\
\hline Terrassa 3 & 4 & 8 & 12 & 33.33 & 66.67 \\
\hline
\end{tabular}

Fuente: elaboración propia. 
Como en el caso de los fiscales, el estudio también ha permitido detectar que existen diferencias notables entre los juzgados de instrucción que actúan en la provincia de Barcelona. Así, como se desprende de la Tabla 8, en los juzgados que resolvieron más de 10 expedientes los porcentajes de denegación del internamiento oscilaron entre el 0 y el $90 \%$. La independencia judicial y las características de cada caso pueden explicar algunas diferencias. No obstante, llama la atención que en ocasiones las diferencias también sean notables entre juzgados de una misma localidad, que se supone que conocen de supuestos similares.

Por lo que se refiere a los argumentos empleados por los jueces para resolver los expedientes de internamiento, cabe recordar que los criterios legales que deben emplearse son el principio de proporcionalidad, el riesgo de incomparecencia, la existencia de actuaciones tendentes a obstaculizar la expulsión, así como la existencia de condenas penales o sanciones administrativas previas o de procedimientos penales o sancionadores pendientes. Pero como se desprende de la Tabla 9 y de forma similar a lo constatado respecto de los fiscales, el estudio ha puesto de relieve que las decisiones judiciales se basan sobre todo en criterios de integración y no de seguridad.

Así, el peso otorgado a los antecedentes policiales y penales es escaso (se mencionan solo en el 25\% de los autos que autorizan el internamiento) a pesar de su profusa invocación en las solicitudes policiales. Para los jueces son mucho más relevantes, en cambio, factores como el arraigo familiar o la existencia de domicilio conocido, argumentos que se emplearon en el 56 y 54\% de los autos que rechazaron el internamiento, y en el 69 y $57 \%$ de los autos que lo autorizaron. También cabe destacar que los medios económicos se aludieron en el $48 \%$ de los autos favorables al internamiento y en el 13\% de los que lo rechazaron, lo cual revela que este argumento juega un papel secundario, pero no despreciable, en las decisiones judiciales. En todo caso, las decisiones judiciales se basan sobre todo en el criterio del riesgo de incomparecencia, que se interpreta desde criterios de integración y no de peligrosidad. 
TABLA 9

MOTIVOS ADUCIDOS POR LOS JUECES DE INSTRUCCIÓN EN RELACIÓN CON LAS SOLICITUDES DE INTERNAMIENTO

\begin{tabular}{|l|c|}
\hline Argumento contrario al internamiento & Porcentaje de utilización \\
\hline Existencia de arraigo familiar & 56,9 \\
\hline Existencia de domicilio estable & 54,1 \\
\hline Existencia de arraigo social & 15,6 \\
\hline Existencia de medios económicos & 13,5 \\
\hline No obstaculización de la expulsión & 7,3 \\
\hline No notificación de la expulsión & 6,6 \\
\hline No firmeza de la expulsión & 5,9 \\
\hline Antecedentes policiales insuficientes & 5,5 \\
\hline Motivos de salud & 2,1 \\
\hline Existencia de autorización de residencia & 1,0 \\
\hline Otros & 26,7 \\
\hline Argumento favorable al internamiento & Porcentaje de utilización \\
\hline Carencia de arraigo familiar & 69,5 \\
\hline Carencia de domicilio estable & $5,3,6$ \\
\hline Carencia de medios económicos & 48,9 \\
\hline Antecedentes penales & 24,6 \\
\hline Antecedentes policiales & \\
\hline Autorizaciones de otros juzgados & \\
\hline Documentación en trámite & \\
\hline Uso de nombre supuesto & \\
\hline Otros & \\
\hline & \\
\hline
\end{tabular}

Fuente: elaboración propia.

\section{CONCLUSIONES}

La práctica del internamiento en la provincia de Barcelona analizada en este trabajo pone de relieve que se trata de una institución en claro retroceso: el número de solicitudes policiales se está reduciendo de forma sensible por diversos factores. En todo caso, la importante 
oposición judicial a dichas solicitudes (alrededor del 50\% en los últimos años) y la escasa eficacia de los internamientos que sí se decretan (algo menos del 50\% de los internos acaban siendo expulsados) están contribuyendo de forma decisiva a la crisis de esta figura.

La investigación también ha puesto de relieve que el internamiento tiene un impacto heterogéneo en las personas extranjeras que están en situación irregular y son susceptibles de ser repatriadas. Cuestiones organizativas como la inexistencia de un módulo de mujeres en el CIE tienen como consecuencia que las solicitudes de internamiento se produzcan casi solo en relación con varones. Factores como la mayor o menor posibilidad de ejecutar una orden de salida obligatoria a determinados países determinan que las posibilidades de ser internado sean bastante distintas en función del país de origen. La aplicación de una medida restrictiva de la libertad como el internamiento depende, pues, en buena medida de factores ajenos al comportamiento y la trayectoria de la persona extranjera.

Aunque el internamiento es una medida cautelar para hacer efectiva una orden de salida obligatoria, el estudio ha puesto de manifiesto que los criterios empleados por los diversos actores en la práctica para fundamentar su posición son diferentes. Las solicitudes policiales se basan sobre todo en los antecedentes policiales y penales, así como en la inexistencia de un domicilio estable, conceptos vinculados sobre todo a la peligrosidad y la seguridad ciudadana. Existe, de hecho, una priorización de las expulsiones que se pretenden ejecutar, que está basada en criterios de seguridad, aunque estos se vinculen a conceptos discutibles como los antecedentes policiales.

En cambio, los informes de los fiscales y las decisiones de los jueces dan más importancia al arraigo familiar del extranjero, a la existencia de un domicilio estable y, en definitiva, a su integración y socialización. Esta importante discrepancia explica en buena medida que la mitad de las solicitudes policiales sean rechazadas en la provincia de Barcelona.

El estudio también ha demostrado que en el seno de todas las instituciones existen diferencias notables al aplicar unos criterios de actuación que en teoría son los mismos. Estas diferencias pueden deberse en muchas ocasiones a las peculiaridades del caso. Pero las diferencias entre los expedientes tramitados por juzgados de una misma localidad ponen de relieve que funcionamiento global del sistema plantea importantes problemas de igualdad y seguridad jurídica. 


\section{LISTA DE REFERENCIAS}

Bainbridge, S. M., y Gulati, G. (2002). How do judges maximize - The same way everybody else does-boundedly: Rules of thumb in securities fraud opinions. Emory Law Journal 51, 83-151.

Barbero, I., Blanco, C., y Arrese, N. (2018). El internamiento de extranjeros: un análisis empírico de los procedimientos instruidos en la Comunidad Autónoma Vasca. Revista Vasca de Administración Pública 110 (I), 17-55.

Bosworth, M. (2014). Inside Immigration detention. Oxford: Oxford University Press.

Boza Martínez, D. (2017). El internamiento de personas extranjeras: más allá de los límites de la privación de libertad. En A. López-Sala y D. Godenau (Coords.), Estados de contención, estados de detención (pp. 97118). Barcelona: Anthropos.

Daunis Rodríguez, A. (2016). Internamiento de extranjeros en España: análisis crítico sobre sus condiciones materiales y tratamiento legal. En P. Laurenzo Copello y A. Daunis Rodríguez (Coords.), Colectivos en los márgenes del Derecho (pp. 137-181). Valencia: Tirant lo Blanch.

De Genova, N., y Pautz, N. (Eds.), (2010). The deportation regime: sovereignity, space and freedom of movement. Durcham, NC: Duke University Press.

Dhami, M. K. (2003). Psychological models of professional decision making. Psychological Science 14(2), 175-180.

Dhami, M. K., y Ayton, P. (2001). Bailing and jailing the fast and frugal way. Journal of Behavioral Decision Making, 14 (2), 141-168.

Fernández Bessa, C., y Brandáriz García, J. A. (2016). Transformaciones de la penalidad migratoria en el contexto de la crisis económica: el giro gerencial del dispositivo de deportación, InDret. Revista para el análisis del Derecho, 4, 1-25.

Fischer, N. (2012). Negotiating Deportations: An Ethnography of the Legal Challenge of Deportation Orders in a French Immigration Detention Centre. En B. Anderson, M. Gibney y E. Paoletti (Eds.). The Social, Political and Historical Contours of Deportation, (pp. 123-142). New York: Springer.

García España, E. (2017). Razones para el cierre de los CIE: del reformismo a la abolición. Observatorio Criminológico del Sistema Penal ante la Inmigración. Málaga: Universidad de Málaga.

Gibney, M. J., y Hansen, R. (2003). Deportation abd the liberal state: the forcible return of asylum seekers and unlawful migrants in Canada, Germany and the United Kingdom. Geneva: UNHCR.

Gigerenzer, G., y Engel, E. (2006). Heuristics and the Law. Cambridge MA: The MIT Press.

Gigerenzer, G., y Goldstein, D. G. (1996). Reasoning the fast and frugal way: models of bounded rationality. Psychological Review 103(4), 650-669.

González Beilfuss, M. (2017). Los CIE: una realidad controvertida y compleja. Anuario CIDOB de la Inmigración 2017, 298-316. 
González Beilfuss, M. (2016). El régimen jurídico de los Centros de internamiento de extranjeros: evolución normativa y cuestiones pendientes. En M. Revenga Sánchez y J. J. Fernández Allés (Coords.), Los centros de internamiento de extranjeros. Régimen jurídico tras el Reglamento de 2014 y la STS de 10 de febrero de 2015 (pp. 37-58) Valencia: Tirant lo Blanch.

Gurhrie C., Rachlinski, J. J., y Wistrich, A. J. (2000). Inside the judicial mind. Cornell Law Review 86, 777-830.

Jarrín Morán, A., Rodríguez García, D. y De Lucas, J. (2012). Los centros de internamiento de extranjeros en España: origen, funcionamiento e implicaciones jurídico-sociales. Documentos CIDOB, Migraciones, 26, Barcelona.

Kalir, B. (2017). State desertion and "out-of-procedure" asylum seekers in the Netherdands. Focaal: Journal of Global and Historical Anthropology 77 (1), 63-75.

Kalir, B., (2015). The Jewish state of anxiety: Between moral obligation and fearism in the treatment of African asylum seekers in Israel. Journal of Ethnic and Migration Studies 41 (4), 580-598.

Kalir, B., y Wissink, L. (2016). The deportation continuum: convergences between state agents and NGO workers in the Dutch deportation field. Citizenship Studies, 20 (1), 34-49.

Kalir B. y Rozakou, K. (2016). "Giving Form to Chaos": The Futility of EU Border Management at Moria Hotspot in Lesvos. Environment and Planning D. Society \& Space (Forum) (http://societyandspace.org/2916)

Larrauri, E. (2016). Antecedentes penales y expulsión de personas inmigrantes. Revista para el análisis del derecho, 2, 1-29.

Leekes, A., y Broeders, D. (2010). A case of mixed motives?: formal and informal functions of administrative immigration detention. British Journal of Criminology, 50 (5), 830-850.

López Benítez, M. (2014). El nuevo régimen jurídico de los centros de internamiento de extranjeros. Revista Vasca de Administración Pública, 99100, 1905-1935.

Martínez Escamilla, M. (2016). Centros de internamiento para extranjeros. Estado de la cuestión y perspectivas de futuro. Revista Electrónica de Ciencia Penal y Criminología 18-23, 1-37. (http://criminet.ugr.es/recpc/18/ recpc18-23.pdf)

Martínez Escamilla, M. (2015). Detención, internamiento y expulsión administrativa de personas extranjeras. Madrid, Repositorio E-Prints Universidad Complutense (http://eprints.ucm.es/34492/)

Poblet, M., y Casanovas, P. (2005). Recruitment, Professional Evaluation and Career of Judges and Prosecutors in Spain, En G. Di Federico (Ed.), Recruitment, professional evaluation and career of judges and prosecutors in Europe: Austria, France, Germany, Italy, The Netherlands and Spain, (pp. 185-213). Bologna: Lo Scarabeo.

Rachlinski, J. J., Wistrich, A. J., y Guthrie, C. (2012). Altering attention in adjudication. UCLA Law Review, 60, 1586-1595. 
Ryo, E. (2016). Detained: A study of immigration bond hearings. Law and Society Review 50 (I), 117-153.

Sainz de la Maza Quintanal, E. (2017). Actores y dinámicas en la puesta en práctica de las deportaciones. En A. López-Sala y D. Godenau (Coords.), Estados de contención, estados de detención, (pp. 144-168) Barcelona: Anthropos.

Sainz de la Maza Quintanal, E. (2015). Última ratio. El proceso de expulsión de los inmigrantes en situación irregular en España. Madrid: Universidad Complutense de Madrid y Fundación Instituto Universitario de Investigación José Ortega y Gasset.

Solanes Corella, A. (2016). Un análisis crítico de los centros de internamiento de extranjeros en España: normativa, realidad y alternativas. Revista Telemática de Filosofía del Derecho, 19, 37-76.

Tomé García, J. (2014). Internamiento preventivo de extranjeros conforme al nuevo reglamento de los CIE. Madrid: Marcial Pons.

Vallbé, J.-J. (2015). Frameworks for Modeling Cognition and Decisions in Institutional Environments. A Data-driven Approach. Heidelberg: Springer.

Welch, M., y Schuster, L. (2005). Detention of asylum seekers in the US, UK, France, Germany and Italy: A critical view of the globalizing culture of control, Criminal Justice 2005, vol. 5, 331-355. 